\title{
Direct Percutaneous Puncture and Embolization of Visceral Pseudoaneurysm: Safety and Clinical Efficacy
}

\author{
Sunil K. Kumar ${ }^{1, \odot}$ \\ G.V. $\operatorname{Rao}^{3}$ \\ ${ }^{1}$ Department of Interventional Radiology, Asian Institute of \\ Gastroenterology, Davanagere, Hyderabad, Telangana, India \\ ${ }^{2}$ Department of Medical Gastroenterology, Asian Institute of \\ Gastroenterology, Hyderabad, Telangana, India \\ ${ }^{3}$ Department of Surgical Gastroenterology, Asian Institute of \\ Gastroenterology, Hyderabad, Telangana, India
}

K.N. Nagbhushan ${ }^{1}$ Nageshwar D. Reddy²

J Clin Interv Radiol ISVIR:2020;4:135-141

\begin{abstract}
Address for correspondence Sunil Kumar K, MD, Department of Interventional Radiology, Asian Institute of Gastroenterology, \# 3000, 6th main, Swamy Vivekananda Extension, Davanagere, Hyderabad, Telangana 500082, India (e-mail: drsunilkumark@yahoo.in).
\end{abstract}

\begin{abstract}
Purpose The aim of the study is to assess the safety and clinical effectiveness of direct percutaneous embolization of visceral artery pseudoaneurysms.

Materials and Methods Retrospective analysis of patients who had undergone direct percutaneous embolization of visceral artery pseudoaneurysms between January 2012 and May 2017 was performed. The study included 26 patients with a mean age of 36 years (range 10-71 years). The indications for direct percutaneous embolization included the inability to catheterize endovascularly $(n=24)$ or prior embolization $(n=2)$ of the feeding artery. Patient demographics, details of the procedure, complications, and outcomes were evaluated. Patients were followed for a mean of 15 months to assess for recurrence of the pseudoaneurysms on ultrasound.

Results Splenic artery $(n=13)$, gastroduodenal artery $(n=4)$, right hepatic artery $(n=3)$, pancreaticoduodenal arteries $(n=3)$, left gastric artery $(n=2)$, and left hepatic artery $(n=1)$ were the embolized arteries. Etiology for pseudoaneurysm were pancreatitis $(n=20)$, trauma $(n=2)$, postoperative $(n=2)$, and few were incidentally detected $(n=2)$. N-butyl cyanoacrylate (NBCA) with lipiodol was used in $23(88.4 \%)$ patients, coil in one (3.8\%), and both coil and NBCA in two patients (7.7\%). Embolization of the pseudoaneurysm was successful in all cases. No procedure-related complication was observed. Follow-up showed no recurrence of the pseudoaneurysm. Self-limiting

\section{Keywords}

- visceral artery pseudoaneurysm

- percutaneous embolization

- gastroduodenal artery splenic infarct was seen in six patients. Self-limiting abdominal pain was seen in all the patients with embolization with NBCA. One patient developed liver infarct and subsequent liver abscess requiring percutaneous drainage.

Conclusion Direct percutaneous embolization is safe and effective in the treatment of visceral artery pseudoaneurysms and should be considered as an alternative in patients with a failed endovascular approach.
\end{abstract}

DOI https://doi.org/ 10.1055/s-0040-1721529 ISSN 2457-0214.
(C) 2020. Indian Society of Vascular and Interventional Radiology. This is an open access article published by Thieme under the terms of the Creative Commons Attribution-NonDerivative-NonCommercial-License, permitting copying and reproduction so long as the original work is given appropriate credit. Contents may not be used for commercial purposes, or adapted, remixed, transformed or built upon. (https://creativecommons.org/licenses/by-nc-nd/4.0/).

Thieme Medical and Scientific Publishers Pvt. Ltd. A-12, 2nd Floor, Sector 2, Noida-201301 UP, India 


\section{Introduction}

Visceral artery aneurysms and pseudoaneurysms arise from the branches of celiac, superior mesenteric, inferior mesenteric, and renal arteries. ${ }^{1}$ Visceral artery aneurysms and pseudoaneurysm are rare causes of gastrointestinal (GI) bleeding with a reported incidence of 0.01 to $0.2 \%$ on autopsy. ${ }^{2}$ The etiology of visceral artery pseudoaneurysms includes infection, trauma, and iatrogenic injuries. Atherosclerosis, fibromuscular dysplasia, or collagen vascular disorders are the causes of true aneurysm. Most of the pseudoaneurysms are asymptomatic and are incidentally detected during evaluation of other conditions. Pseudoaneurysms can rupture and present as upper or lower GI bleed and hematuria. ${ }^{1-6}$ The incidence of rupture of pseudoaneurysms can range from 2 to $80 \%$ depending on the location with mortality rates as high as $100 \% .{ }^{4,5}$ While the true aneurysms smaller than $2 \mathrm{~cm}$ can be monitored, the pseudoaneurysms need to be treated irrespective of their size. ${ }^{1,4}$ Treatment options for pseudoaneurysms include endovascular management and surgery, endovascular embolization, or stent graft placement are usually the first line of therapies. ${ }^{6}$

Percutaneous embolization of pseudoaneurysms for difficult or inaccessible anatomical sites has been mentioned in a few case reports and case series in the literature. The largest case series included 21 patients. ${ }^{7-19}$ Previous studies are limited by small size of the study population and lack of durable follow-up. In this study, we would like to assess the safety and clinical effectiveness of the percutaneous embolization in the management of visceral artery pseudoaneurysms in a large group of patients.

\section{Materials and Methods}

A retrospective analysis of patients who had undergone percutaneous embolization for visceral artery pseudoaneurysm at our tertiary care center between January 2012 and May 2017 was performed. Ethical review was not required for this retrospective study. The indications for direct percutaneous embolization included failure of selective catheterization of the feeding artery supplying the pseudoaneurysm or prior embolization of the proximal segment of the feeding artery.

A total of 26 patients (19 men and 7 women) with a mean age of 36 years (range 10-71 years) were included in this study. Patient demographics, details of percutaneous embolization, technical success, procedure-related complications, and outcomes were evaluated. Patients were followed for a mean 15 months (12-18 months) by ultrasound to assess for recurrence of pseudoaneurysms.

\section{Angiography and Endovascular Technique}

Computed tomographic (CT) angiography of the abdomen and pelvis was obtained on a six-slice multidetector CT scanner (Siemens Emotion, Siemens Healthcare $\mathrm{GmbH}$ ). Angiography was performed on single plane digital subtraction angiography (DSA) system (Axiom Artis U, Siemens Healthcare
$\mathrm{GmbH})$. Angiography and endovascular interventions were performed through a right transfemoral arterial access. Arterial access was obtained through right common femoral artery and a $6 \mathrm{~F}$ vascular sheath (Cordis) was placed. Using a 5F Simmons angiography catheter (Cook Medical) and 0.035inch hydrophilic guidewire (Radifocus, Terumo), the celiac trunk, superior mesenteric artery, and inferior mesenteric artery were catheterized. Superselective catheterization of the common hepatic artery, gastroduodenal artery (GDA), left gastric artery (LGA), and splenic artery were performed using a $4 \mathrm{~F}$ Kumpe/Multipurpose catheter or a 3F microcatheter (Cook Medical). DSA images were reviewed for presence of pseudoaneurysm. In 24 patients, the feeding artery was not accessible for selective catheterization and two patients had prior embolization of the feeding artery.

For percutaneous embolization, the pseudoaneurysms were accessed percutaneously using an $18-G$ spinal needle (Meditop Corporation) under ultrasound guidance. Percutaneous angiography was performed through needle, to assess the size of pseudoaneurysm, feeding artery, and flow dynamics. Embolization was performed by using either $\mathrm{N}$-butyl cyanoacrylate (NBCA) (Xion, Reevax Pharma) with lipiodol (Guerbet) or coil (Cook Medical) or both under ultrasound and fluoroscopic guidance. The NBCA and lipiodol were mixed in the ratio of $1: 3,1: 2$, and $1: 1$ depending upon the flow dynamics. A total of 0.035 compatible coils of different sizes were used ( $3-30 \mathrm{~mm}, 3-40 \mathrm{~mm}$, and $3-50 \mathrm{~mm}$ ). The average fluoroscopy time was 3 minutes. A repeat catheter angiography was performed to confirm the complete occlusion of the pseudoaneurysm.

Technical success was defined as complete occlusion of pseudoaneurysm at the end of procedure confirmed by catheter angiography. All patients were followed for mean of 15 months by ultrasound to assess for recurrence of pseudoaneurysm. First ultrasound was done after 1 month and subsequent follow-up was done at interval of 3 months.

\section{Results}

Patient demographics, etiology of pseudoaneurysms, and the arteries involved are summarized in - Table $\mathbf{1}$. The underlying cause of the pseudoaneurysm was pancreatitis in 20 patients, trauma in two patient, iatrogenic injury in two patients, and incidentally detected pseudoaneurysm in two patients. CT angiography was positive in all cases. Average size of pseudoaneurysm was $15 \mathrm{~mm}$ (range 10-30 mm)

A failed selective catheterization was the indication for percutaneous embolization in 24 patients (-Figs. 1-3). A prior embolization of the feeding artery in two patients ( - Fig. 4) precluded endovascular embolization. Splenic artery was the most common vessel involved $(n=13)$ followed by the GDA $(n=4)$, pancreaticoduodenal arteries $(n=3)$, right hepatic artery $(n=3), \operatorname{LGA}(n=2)$, and left hepatic artery $(n=1)$.

Technical details, outcome, and complications are summarized in - Table 2. Embolization was performed using NBCA and lipiodol combination in 23 patients (88.4\%; - Figs. 1-4), coil in one patient (3.8\%), and both coil and glue in two (7.7\%) 
patients ( - Fig. 5). Embolization of the pseudoaneurysm was successful in all the cases. None of the patients had immediate procedure-related complications. Minimal flow of NBCA into nontarget vessel was seen in all the patients with NBCA embolization.

Out of 13 patients with splenic artery pseudoaneurysms, six patients developed self-limiting splenic infarcts. One patient with hepatic artery embolization developed infarction and subsequent abscess requiring percutaneous

Table 1 Showing patients demographics, etiology, and arteries involved

\begin{tabular}{|l|l|}
\hline Number of patients & 26 \\
\hline M:F & $19: 7$ \\
\hline Age, mean & $36(10-71 \mathrm{y})$ \\
\hline Etiology & \\
\hline Pancreatitis & 20 \\
\hline Trauma & 2 \\
\hline Postoperative & 2 \\
\hline Incidental & 2 \\
\hline Arteries involved & \\
\hline Splenic artery & 13 \\
\hline Gastroduodenal artery & 4 \\
\hline Right hepatic artery & 3 \\
\hline Left hepatic artery & 1 \\
\hline Left gastric artery & 2 \\
\hline Pancreaticoduodenal arteries & 3 \\
\hline
\end{tabular}

drainage. Self-limiting abdominal pain was seen in all the patients with NBCA embolization immediately after the procedure.

Follow-up ultrasound at a mean 15-month period showed no residual or recurrence of pseudoaneurysm.

\section{Discussion}

Visceral artery aneurysms and pseudoaneurysms arise from the branches of celiac, superior mesenteric, inferior mesenteric, or renal arteries. ${ }^{1}$ Focal discontinuity in vessel wall by infection, inflammation, trauma, and iatrogenic injury will lead to extravasation of blood contained by the vessel wall or by the adjacent soft-tissue, leading to the formation of pseudoaneurysm. ${ }^{2,3}$ The incidence of rupture of pseudoaneurysms varies from 2 to $80 \%$ with untreated mortality rates reaching up to $100 \%{ }^{5}$ Most of the pseudoaneurysms are asymptomatic and incidentally detected on imaging during evaluation of other conditions. ${ }^{3}$

Noninvasive evaluation of visceral artery aneurysms and pseudoaneurysms is performed with ultrasound, CT angiography, and magnetic resonance angiography. Pseudoaneurysm is seen as an outpouching from the vessel wall with swirling motion and to-and-fro waveform on the Doppler. In CT pseudoaneurysm is seen as low attenuation rounded focus adjacent to artery in plain CT. High attenuation adjacent to the pseudoaneurysm indicates pseudoaneurysm rupture. Contrast study shows enhancement similar to adjacent artery in both arterial and venous phase. Convention angiography is the gold standard for the diagnosis of pseudoaneurysms and
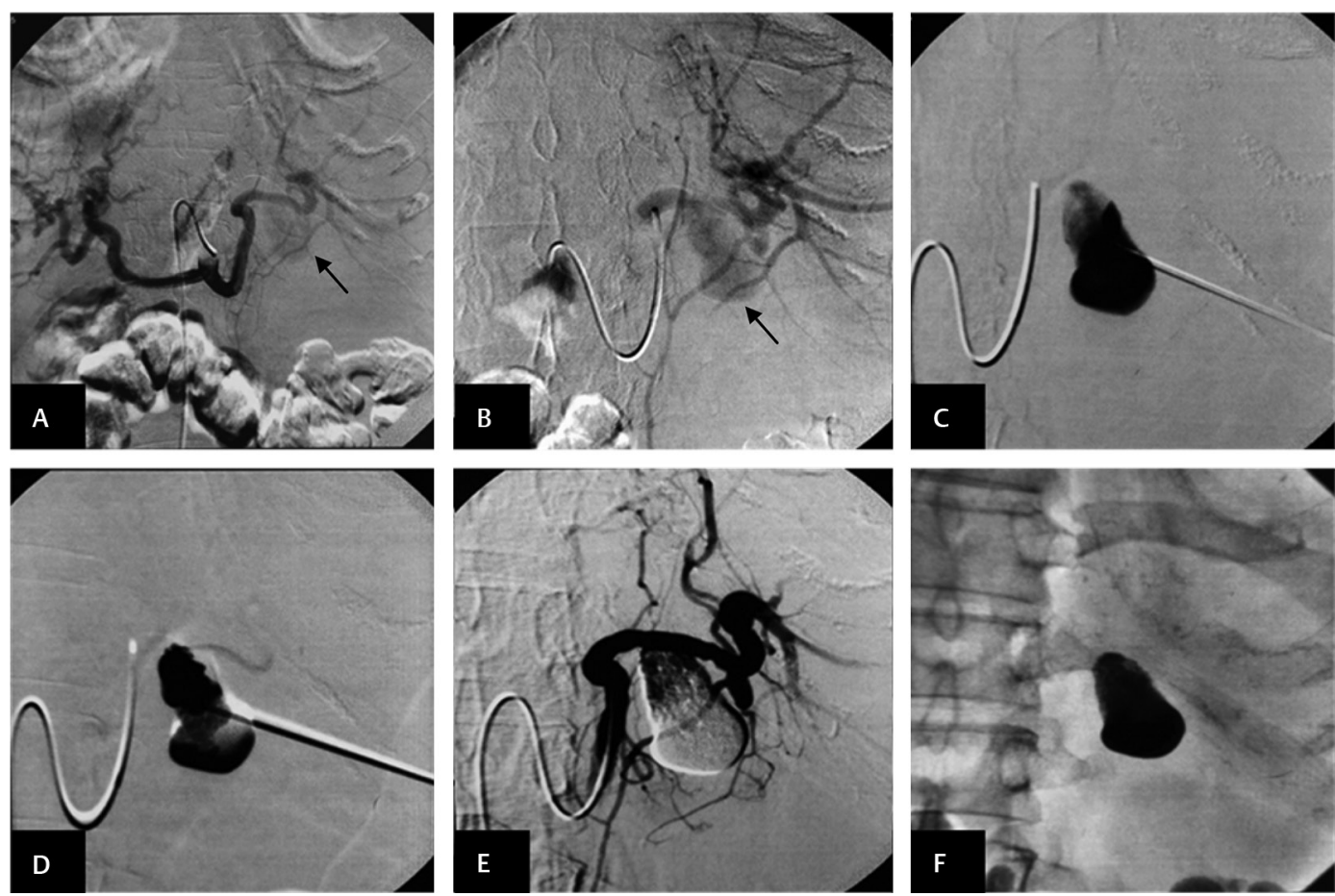

Fig. 1 A 38-year-old male patient with history of chronic pancreatitis and splenic artery pseudoaneurysm. (A, B) Convention angiography shows a splenic artery pseudoaneurysm (black arrows). Transcatheter embolization failed due to difficult access. (C) Percutaneous puncture and angiography were performed to confirm the position of the needle. (D) Embolization with NBCA and lipiodol was performed. (E) Postembolization angiography shows no filling of the pseudoaneurysm. (F) Postprocedure fluoroscopy image shows lipiodol cast in the pseudoaneurysm. NBCA, N-butyl cyanoacrylate. 

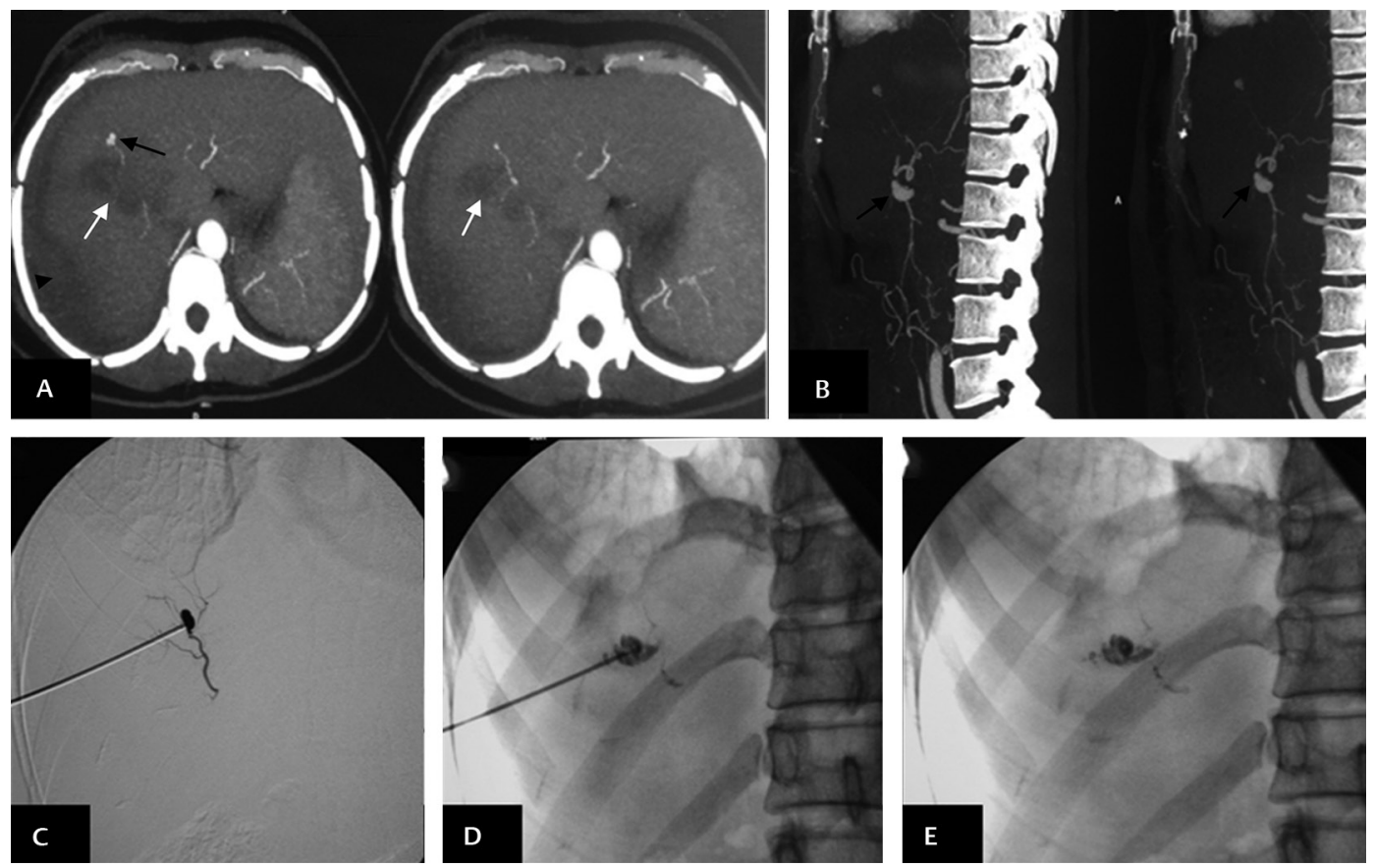

Fig. 2 A 38-year-old female patient with history of trauma and liver laceration. CT (A) axial and (B) oblique sagittal MIP images show a liver laceration (white arrows) and subcapsular hematoma (arrowhead) with a pseudoaneurysm (black arrows) arising from the right hepatic artery. Convention embolization failed due to difficult arterial access. (C) Percutaneous puncture and angiography confirmed the pseudoaneurysm. (D) This was followed by embolization of the pseudoaneurysm with NBCA and lipiodol. (E) Mild reflux of NBCA into right hepatic artery is noted. $\mathrm{CT}$, computed tomography; MIP, maximum intensity projection; NBCA, N-butyl cyanoacrylate.
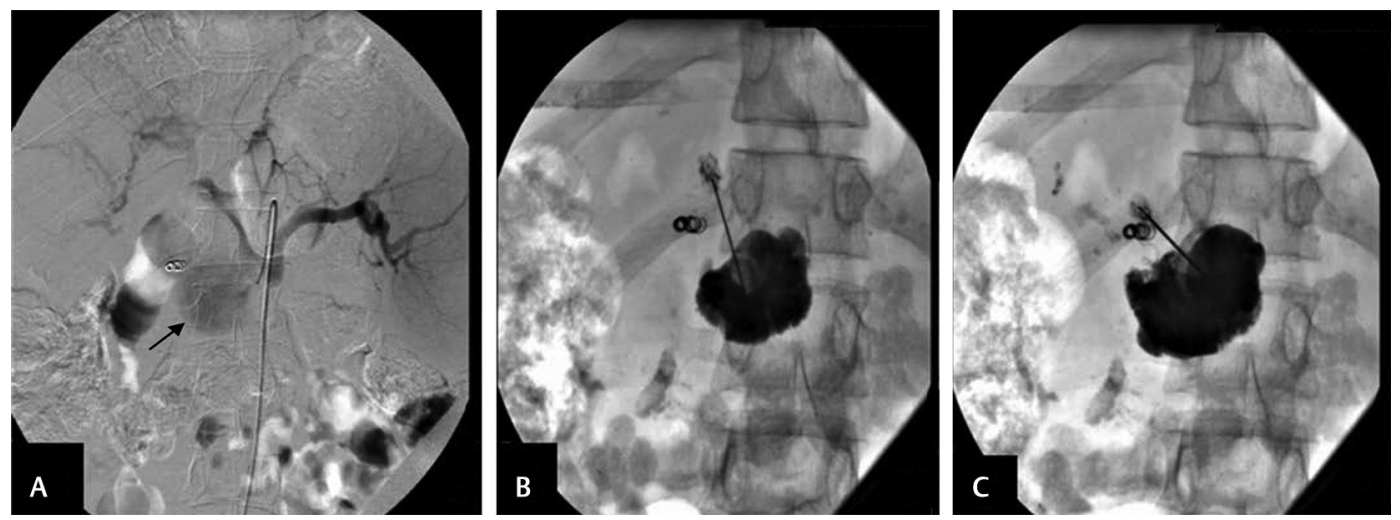

Fig. 3 A 21-year-old male patient with chronic pancreatitis and prior embolization of right hepatic artery. (a) Convention angiogram showed large pseudoaneurysm from proximal gastroduodenal artery (black arrow). Transcatheter embolization failed due to difficult access. (b) Percutaneous puncture and angiography confirmed the position of the needle. (c) Embolization was performed with NBCA and lipiodol. NBCA, N-butyl cyanoacrylate.

has the advantage of real-time assessment of vascular bed including identification of feeding vessel, collateral vessel, and concomitant therapeutic intervention ${ }^{3,5,6}$ In the present study, pseudoaneurysms were detected by CT angiography and were identifiable on Doppler and conventional angiography in all cases.

Treatment options for visceral artery pseudoaneurysms include endovascular embolization, stent graft placement, and surgery. Endovascular embolization and stent graft placement are recommended as the first-line therapy. Surgical management of pseudoaneurysms includes resection with a bypass, arterial ligation, and partial or complete organ removal. Surgical treatment is invasive and is often associated with significantly higher morbidity and mortality rates. ${ }^{3,6,20}$ Success rate of transcatheter embolization for both aneurysms and pseudoaneurysms is high (98\%) with 30-day mortality rate of $3.4 \%{ }^{9}$

Direct percutaneous approach was first described by Cope and Zeit in 1986 for the treatment of a femoral pseudoaneurysm. ${ }^{21}$ Percutaneous embolization can be performed in pseudoaneurysms with difficult anatomy, inaccessible sites, prior embolization of feeding vessel, and in whom feeding artery cannot be identified on angiography. ${ }^{9-15}$ Available 

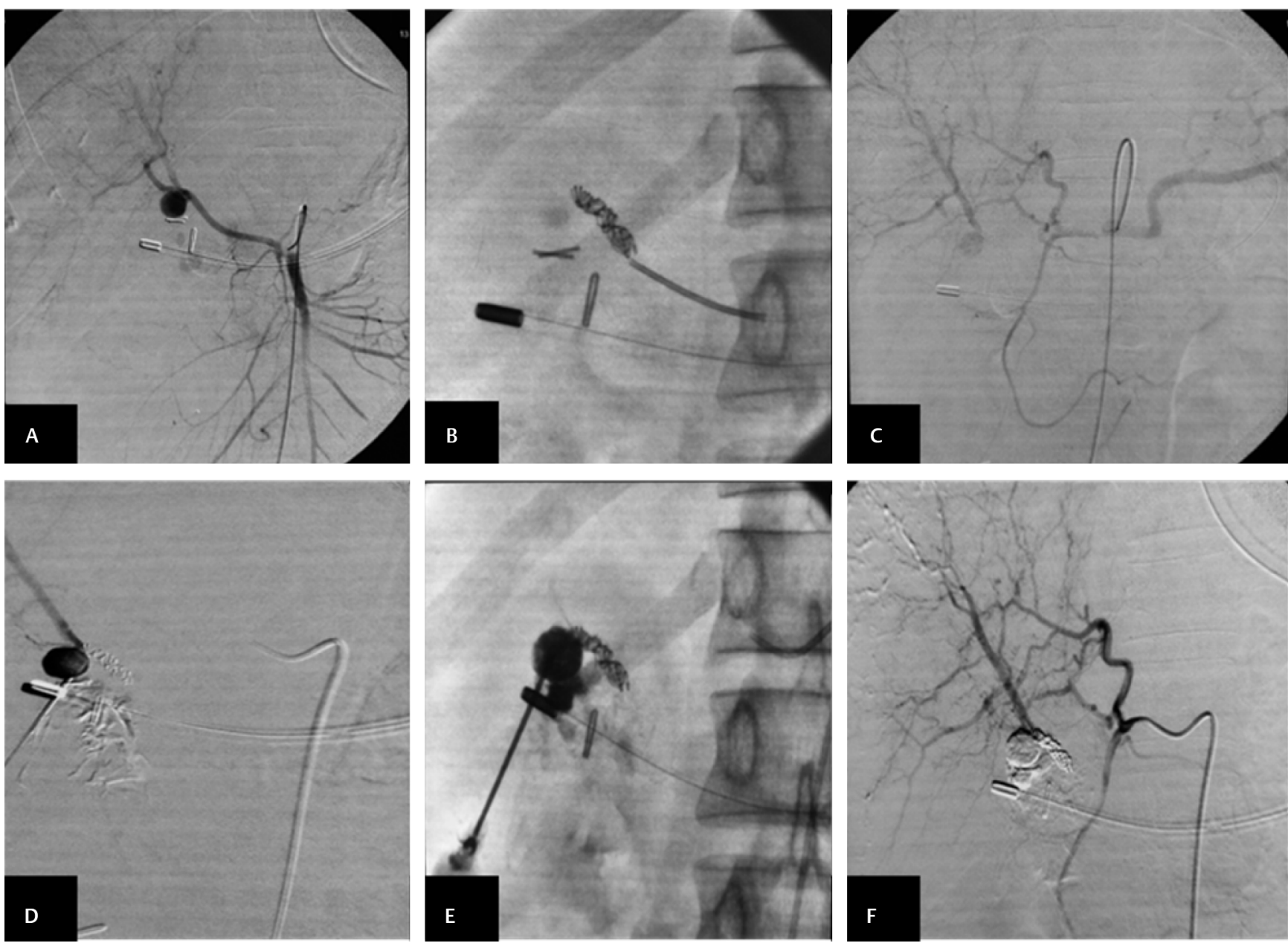

Fig. 4 A 26-year-old female patient with history of cholecystectomy and pain abdomen. (A) Convention angiogram showing pseudoaneurysm arising from the right hepatic artery. (B) Coil embolization of right hepatic artery was performed. (C) Postembolization angiography showed persistent filling of the pseudoaneurysm. Further endovascular management was not feasible due to occlusion of proximal vessel by coils. (D) Percutaneous puncture and angiography confirmed the position of the needle. (E) Embolization with NBCA and lipiodol was performed.

(F) Postembolization angiography showed no filling of the pseudoaneurysm. NBCA, N-butyl cyanoacrylate.

Table 2 Showing technical details, outcome, and complications

\begin{tabular}{|l|l|}
\hline Embolization & 23 \\
\hline NBCA and lipiodol & 1 \\
\hline Coil & 2 \\
\hline Both & $26(100 \%)$ \\
\hline Outcome & Nil \\
\hline Technical success & \multicolumn{2}{|l|}{} \\
\hline Recurrence of pseudoaneurysm & 25 \\
\hline Complications & 6 \\
\hline Self-limiting abdominal pain & 1 \\
\hline Splenic infarct & \\
\hline Liver abscess &
\end{tabular}

Abbreviation: NBCA, N-butyl cyanoacrylate.

literature on percutaneous embolization of visceral artery pseudoaneurysms has been summarized in -Table $\mathbf{3}$. Various embolic materials have been used for percutaneous embolization. Most commonly used embolic material was thrombin, followed by NBCA and coils. In the present study, a combination of NBCA and lipiodol was used in 23 patients (88.4\%), coil in one patient (3.8\%), and both coil and NBCA in two patients $(7.7 \%)$. Use of thrombin has been associated with recurrence of pseudoaneurysm in significant number of cases requiring reintervention. Coils and NBCA are not associated with recurrence or reformation of pseudoaneurysm..$^{7-9,16-18}$ None of the patients in our study had residual or recurrence of pseudoaneurysm.

Percutaneous embolization has been associated with a few complications. There are no reports of procedural complications such as rupture of the pseudoaneurysm. Jejunal stricture has been reported by Gulati et al due to reflux of NBCA into jejunal branches ${ }^{7-9,11-19}$ Most common complication in our study was self-limiting splenic infarct (six patients) due to the reflux of NBCA into distal splenic branches. Self-limiting abdominal pain was seen in all the patients with glue embolization. One patient with hepatic artery embolization developed liver infarct and abscess which was managed with percutaneous drainage.

Our study has many limitations. Despite being one of the largest studies, our study included only 26 patients, and is 

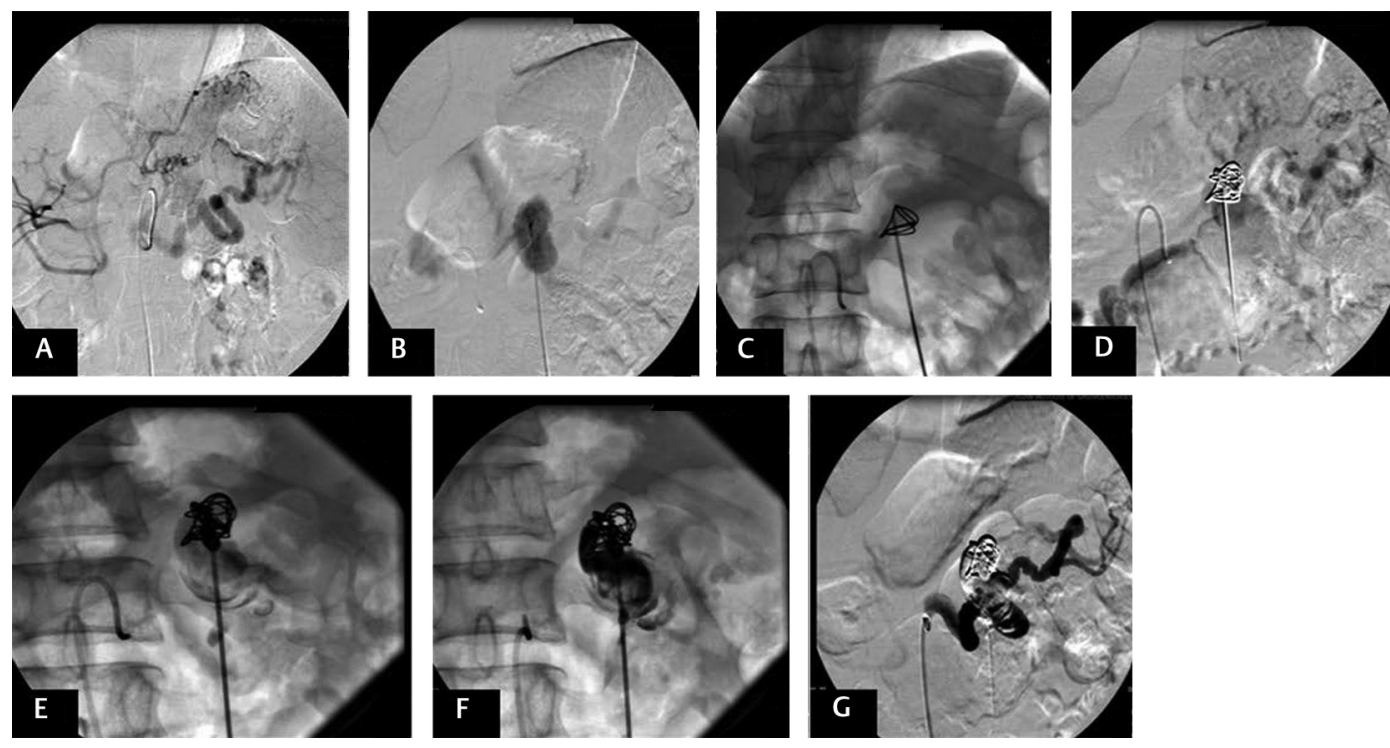

Fig. 5 A 58-year-old male patient with chronic pancreatitis and splenic artery pseudoaneurysm. (A, B) Convention angiogram shows splenic artery pseudoaneurysm. Transcatheter embolization failed due to difficult access. (C) Percutaneous puncture and angiography confirmed the position of the needle. (C, D) Embolization was performed with coils. (E) Postembolization angiography showed persistent filling of pseudoaneurysm. (F) Further embolization was performed with NBCA and lipiodol. (G) Postembolization angiography showed no filling of the pseudoaneurysm.

Table 3 Summary of literature on percutaneous embolization of pseudoaneurysm

\begin{tabular}{|l|l|l|l|l|}
\hline & No. of cases & Embolic agent & Reintervention & Complications \\
\hline Gorsi et al & 21 & NBCA & - & Pneumothorax \\
\hline Fankhauser et al & 2 & Coil & - & - \\
& 3 & Thrombin & 1 & - \\
\hline Vyas et al $^{8}$ & 3 & NBCA & - & - \\
\hline Yadav et al $^{12}$ & 2 & Thrombin & 1 & - \\
\hline Nicholson et al $^{16}$ & 4 & NBCA & - & - \\
\hline Laganà et al & 4 & Thrombin & 4 & - \\
\hline Kuno et al & 2 & Thrombin & 2 & - \\
\hline Ghassemi et al ${ }^{14}$ & 1 & Coil & - & - \\
\hline Ward et al ${ }^{13}$ & 1 & Thrombin & - & - \\
\hline Santiagu et al ${ }^{15}$ & 1 & Thrombin & - & - \\
\hline Puri et al ${ }^{18}$ & 1 & Thrombin & - & - \\
\hline Barbiero et al ${ }^{11}$ & 1 & Thrombin & 1 & - \\
\hline Gulati et al ${ }^{19}$ & 1 & Thrombin & - & - \\
\hline
\end{tabular}

Abbreviation: NBCA, N-butyl cyanoacrylate.

retrospective in nature. The follow-up of our patients was limited to ultrasound examination only. Additionally, the follow-up period was limited to a mean of 15 months with no regular imaging follow-up after. A large prospective study is required to validate the safety and efficacy of the procedure and the results of our study.

Direct percutaneous embolization is safe and effective in the treatment of visceral artery pseudoaneurysms. It can be considered as an alternative following a failed endovascular approach or when an endovascular approach is not feasible.

\section{Conflicts of Interest}

There was no conflict of interest.

\section{References}

1 Belli AM, Markose G, Morgan R. The role of interventional radiology in the management of abdominal visceral artery aneurysms. Cardiovasc Intervent Radiol 2012;35(2): 234-243

2 Chadha M, Ahuja C. Visceral artery aneurysms: diagnosis and percutaneousmanagement.SeminInterventRadiol2009;26(3): 196-206 
3 Saad NE, Saad WE, Davies MG, Waldman DL, Fultz PJ, Rubens DJ. Pseudoaneurysms and the role of minimally invasive techniques in their management. Radiographics 2005;25 (suppl 1) :S173-S189

4 Loffroy R, Favelier S, Pottecher P, et al. Endovascular management of visceral artery aneurysms: when to watch, when to intervene? World J Radiol 2015;7(7):143-148

5 Madhusudhan KS, Venkatesh HA, Gamanagatti S, Garg P, Srivastava DN. Interventional radiology in the management of visceral artery pseudoaneurysms: a review of techniques and embolic materials. Korean J Radiol 2016;17(3):351-363

6 Jesinger RA, Thoreson AA, Lamba R. Abdominal and pelvic aneurysms and pseudoaneurysms: imaging review with clinical, radiologic, and treatment correlation. Radiographics 2013;33(3):E71-E96

7 GorsiU, Chaluvashetty S, Kalra N, et al. Percutaneous glue embolization as a primary treatment for visceral pseudoaneurysms. Minim Invasive Ther Allied Technol 2020;29(3):170-176

8 Vyas S, Khandelwal N, Gupta V, et al. Minimally invasive image-guided interventional management of hepatic artery pseudoaneurysms. Trop Gastroenterol 2014;35(4):238-245

9 Fankhauser GT, Stone WM, Naidu SG, et al; Mayo Vascular Research Center Consortium. The minimally invasive management of visceral artery aneurysms and pseudoaneurysms. J Vasc Surg 2011;53(4):966-970

10 Kuno RC, Althaus SJ, Glickerman DJ. Direct percutaneous coil and ethanol embolization of a celiac artery pseudoaneurysm. J Vasc Interv Radiol 1995;6(3):357-360

11 Barbiero G, Battistel M, Susac A, Miotto D. Percutaneous thrombinembolization ofa pancreatico-duodenal artery pseudoaneurysm after failing of the endovascular treatment. World J Radiol 2014;6(8):629-635

12 Yadav RR, Boruah DK, Bhattacharyya V, et al. Percutaneous direct needle puncture and transcatheter N-butyl cyanoacrylate injection techniques for the embolization of pseudoaneurysms and aneurysms of arteries supplying the hepato-pancreato-biliary system and gastrointestinal tract. J Clin Imaging Sci 2016;6:48
13 Ward EV, Buckley O, Doody O, Govender P, Conlon K, Torreggiani WC. Percutaneous thrombin embolization of a ruptured peripancreatic pseudoaneurysm. Digestion 2007; 76(3-4):188-191

14 Ghassemi A, Javit D, Dillon EH. Thrombin injection of a pancreaticoduodenal artery pseudoaneurysm after failed attempts at transcatheter embolization. J Vasc Surg 2006;43(3):618-622

15 Santiagu S, Gananadha S, Harrington TJ, Samra JS. Direct percutaneous puncture embolization of a peripancreatic pseudoaneurysm presenting with haemosuccus pancreaticus. J Med Imaging Radiat Oncol 2008;52(4):370-373

16 Nicholson AA, Patel J, McPherson S, Shaw DR, Kessel D. Endovascular treatment of visceral aneurysms associated with pancreatitis and a suggested classification with therapeutic implications. J Vasc Interv Radiol 2006;17(8):1279-1285

17 Laganà D, Carrafiello G, Mangini M, et al. Multimodal approach to endovascular treatment of visceral artery aneurysms and pseudoaneurysms. Eur J Radiol 2006;59(1):104-111

18 Puri S, Nicholson AA, Breen DJ. Percutaneous thrombin injection for the treatment of a post-pancreatitis pseudoaneurysm. Eur Radiol 2003;13(suppl 4) :L79-L82

19 Gulati GS, Gulati MS, Makharia G, et al. Percutaneous glue embolization of a visceral artery pseudoaneurysm in a case of sickle cell anemia. Cardiovasc Intervent Radiol 2006;29(4):665-668

20 Tulsyan N, Kashyap VS, Greenberg RK, et al. The endovascular management of visceral artery aneurysms and pseudoaneurysms. J Vasc Surg 2007;45(2):276-283, discussion 283

21 Cope C,Zeit R. Coagulation of aneurysms by direct percutaneous thrombin injection. AJR Am J Roentgenol 1986;147(2):383-387 